\title{
Granular dynamics simulations of two-dimensional heap formation
}

\author{
J. Baxter, U. Tüzün, and J. Burnell* \\ Department of Chemical and Process Engineering, University of Surrey, Guildford, Surrey, GU2 5XH, United Kingdom \\ D. M. Heyes \\ Department of Chemistry, University of Surrey, Guildford, Surrey GU2 5XH, United Kingdom
}

(Received 12 August 1996)

\begin{abstract}
Granular dynamics simulations have been carried out of vertical feed two-dimensional heap formation by a freefall method using a more realistic granule interaction law than has been employed in previous studies to permit prolonged contacts between adjacent granules. Stable heaps are found to form only on a geometrically rough base comprised of discrete particles, and heap formation is only weakly sensitive to the value of the contact friction coefficient. The appearance of avalanches, the pressure distribution on the base, and the voidage distribution are sensitive to the analytic form of the elastic component of the normal interaction, with a soft-sphere $r^{-36}$ potential giving more realistic behavior than an equivalent Hooke law interaction with the same apparent spring constant. The $r^{-36}$ interaction gives more realistic assembly dynamics as it introduces medium range collective motion caused by particle roughness and shape found in typical granular materials, without having to model anisotropic particles. [S1063-651X(97)02402-1]

PACS number(s): 46.10.+z, 62.20. $-\mathrm{x}, 83.70 . \mathrm{Fn}$
\end{abstract}

\section{INTRODUCTION}

The storage and conveying of granular materials in stockpiles ("heaps") are common industrial practice in, for example, the agricultural, food, coal, cement, and pharmaceutical industries. Product quality, which depends on the dynamics of formation and internal stresses within the heap, is difficult to determine experimentally. Design criteria for the storage and handling of industrial bulk solids have been addressed traditionally using empirical approaches, which have limited predictive capacity beyond a narrow range of external parameters as they are not sufficiently discriminating at a microstructural level. An improved understanding of the formation mechanism and internal state of a heap is therefore a desirable aim. This requires a description of the system at the level of the individual particles. Granular dynamics (GD) is an appropriate computer simulation technique to consider these fundamental issues, as the assembly properties are generated from the specific interactions between the individual grains. The technique has been applied to a wide range of granular unit operations, such as quasistatic flow in hoppers $[1,2]$ and flow down inclined chutes [3-5]. These simulations have shown that specific forces that represent the mechanics of the assembly are important in accounting for observed behavior (e.g., rupture zones in hoppers). Although cellular automata lattice treatments of "sandpile" formation have become popular in recent years [6], they are of limited use in addressing the above issues because the length scale is indeterminate and consequently the approach is too generic to account for the engineering physics of real sandpiles. As will be revealed, the dynamics of granules in a heap spans a wide range of different classes,

\footnotetext{
*Permanent address: New Zealand Institute for Industrial Research and Development, P.O. Box 31-310, Lower Hutt, New Zealand.
}

from lean-phase highly agitated dynamics in the boundary region to slow highly cooperative quasistatic flow further inside the pile, in which contacts between the granules persist for considerable lengths of time. It is important therefore to use equations of motion that can realistically encompass the transition between these various regimes. In this paper we use discrete particle simulation to explore some of the issues relating to stable and realistic heap formation.

\section{THEORY}

Discrete particle computer simulations have been used to make free-standing heaps by a process of continuous random vertical pouring of up to about 1000 two-dimensional particles onto a horizontal substrate. The molecular-dynamics simulation procedure we used involves integrating the classical equations of motion of the disks by a time stepping algorithm due to Verlet [7]. The main difference between the dynamics of granules and molecules is that the force field between the particles has frictional- and velocity-dependent components. Body gravitational forces largely determine the dynamical and rest state of the system. It is important to choose functional forms of the interactions that capture the physics at the assembly level. There is also an elastic normal or excluded-volume interaction that prevents excessive overlap of the model granules. As will be shown, the analytic form of this interaction has a pronounced effect on the dynamics of granular systems and it is important to choose a form that captures the physics of heaps on the length and time scale of the assembly as a whole.

Our previous simulations on hoppers suggest that a force between two model granules separated by center-to-center distance $r$ with the analytic form $F_{e}^{N}(r)=m g(\sigma / r)^{-(n+1)}$, where $n=36$ and $g$ is the gravitational acceleration, produces realistic realizations of hopper flow and internal structure. The corresponding interaction potential is $\phi(r)$ $=m g(\sigma / r)^{-n} / n$. Beyond a separation of $\sigma$ (the nominal par- 


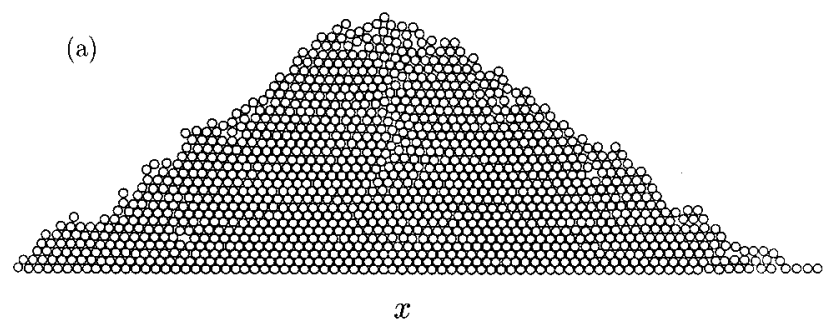

(b)

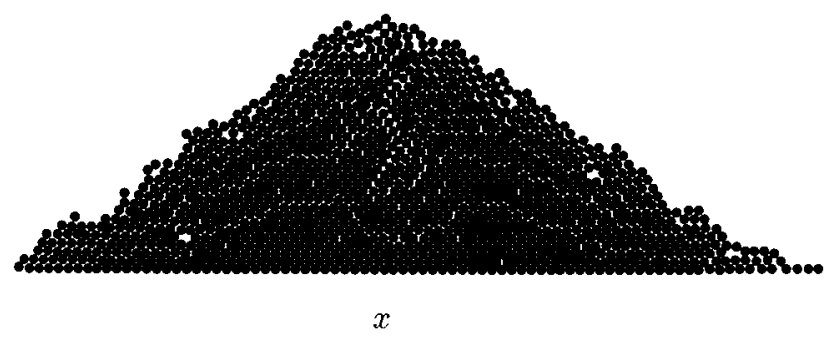

FIG. 1. Snapshot of a heap of 1334 monodisperse particles at near rest after $2.15 \times 10^{3}$ time units. Key: (a) open circles and (b) closed circles to highlight better the dislocations. We have used $n=36$ and $r_{0}=1.2 \sigma$ for $F_{e}^{N}$.

ticle diameter) the force decays slowly to zero. This force law has the useful feature that two particles vertically separated under gravity come to equilibrium at a separation of $r=\sigma$. The force is truncated at a separation of $r_{0}>\sigma$ and is continuously varying with overlap in a way that accounts for the engagement of rough and shaped particles in the normal direction (it can be considered to be equivalent to an $r$-dependent Hooke law spring constant). Also the slow decay of the force beyond $r=\sigma$ also allows for a corresponding gradual tangential ("frictional") interaction. It is clear that assembly frictional interactions extend far beyond the interaction of two surfaces at the nominal physical boundaries of the particles. Such assembly frictional work is required to dilate the assembly around each particle to sustain bulk shear. This is believed to be the physical mechanism that gives rise to the clear long-range connectivity of bulk shear phenomena in granular media (e.g., rupture zones). It has been shown previously that such shear banding was considerably enhanced by increasing $r_{0}$ from $1.034 \sigma$ to $1.2 \sigma$ [1]. In contrast, interactions that have an abrupt cutoff at $r=\sigma$ (e.g., the Hooke law form) are found to yield largely isolated pairwise interactions between the assembly particles with little apparent long-range connectivity.

It is important to emphasize that no attempt is being made in the present study to represent so-called microcontact friction, which operates between asperities on the micrometer distance scale, which could be represented by a Hertzian interaction (the solution for two ideal elastic spherical bodies pressed together). While this might perhaps be appropriate for idealized near-spherical elastic particles under large loads, as is the situation in compaction, the poorly defined (a)

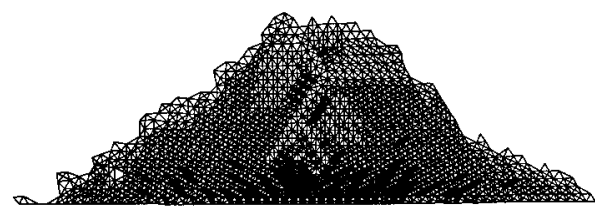

(b)

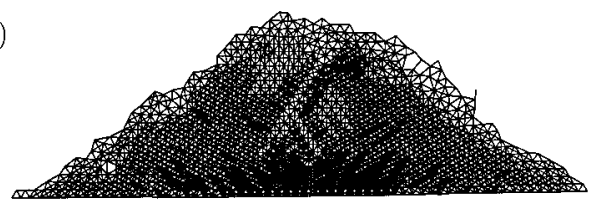

(c)
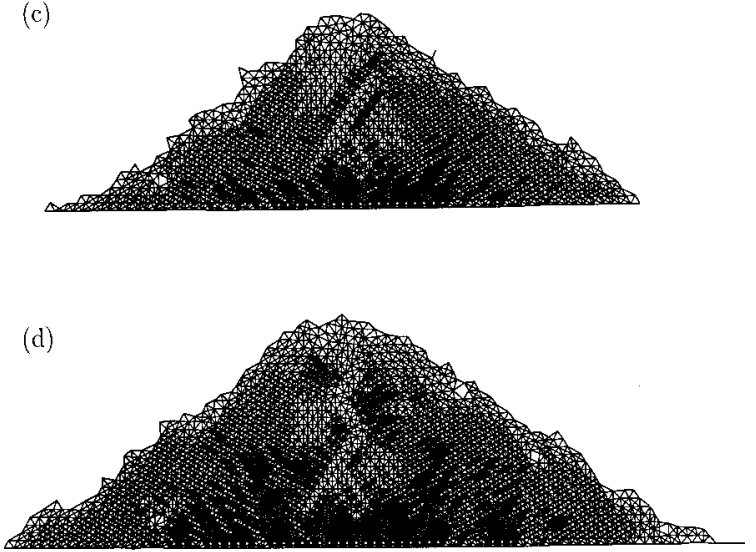

FIG. 2. Density distribution of an evolving heap of monodisperse particles, indicated by center-to-center vectors. Key: (a) before an avalanche at $1.40 \times 10^{3}$ time units, when 869 particles had been deposited; (b) during an avalanche at $1.45 \times 10^{3}$ time units, when 900 particles had been deposited; (c) after avalanche at $1.50 \times 10^{3}$ time units, when 931 particles had been deposited; and (d) for a near-stable state at $2.15 \times 10^{3}$ time units, when 1334 particles had been deposited (same configuration as for Fig. 1). In the interaction, $n=36$ and $r_{0}=1.2 \sigma$.

surface roughness of typical granules, the relatively low loads encountered in hoppers and heaps, and the abovementioned importance of assembly-scale interactions invalidate such a model. This strategy is supported by the experimentally observed fact that the physical behavior in these situations is insensitive to the material constants characterizing the granules: the material constants (e.g., elastic moduli) do not enter design criteria for hoppers, for example. Previous simulations of granular dynamics have adopted a frictional interaction based on the tangential component of the instantaneous relative velocity $\dot{\mathbf{r}}_{i j}$ of the two particles $i$ and $j$ $[4,8]$. However, this prescription inadequately represents the endurance of contacts. In such a model two particles in physical contact will experience a change in the direction of the frictional force when the tangential component of the relative velocity changes sign, as it will do many times in a real system, while still maintaining physical contact. (This phenomenon is called microslip [9].) This does not reflect adequately the duration and buildup of contact deformation. Rather, in our model the frictional force is based on the accumulation of a separate contact displacement $\delta_{F}$, which is incremented for each contact as the assembly evolves. The 
(a)

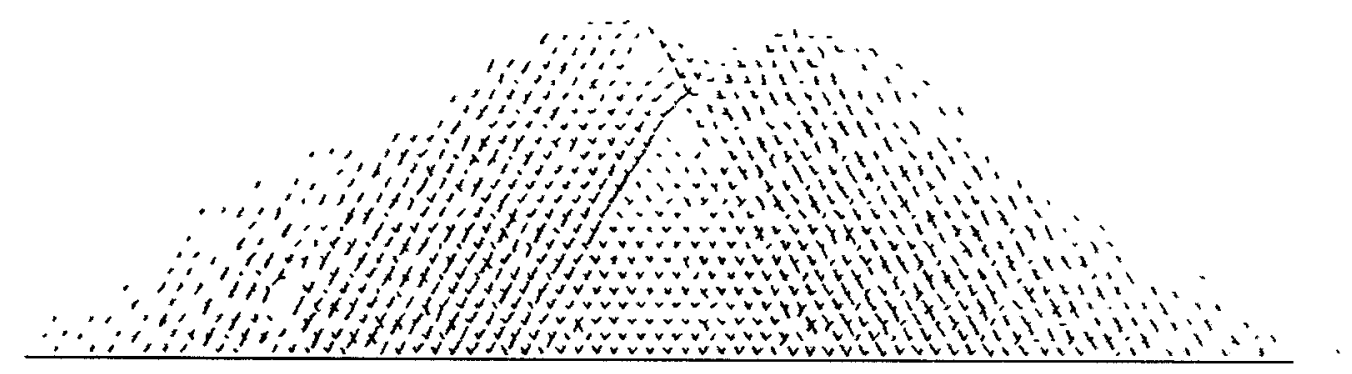

(b)

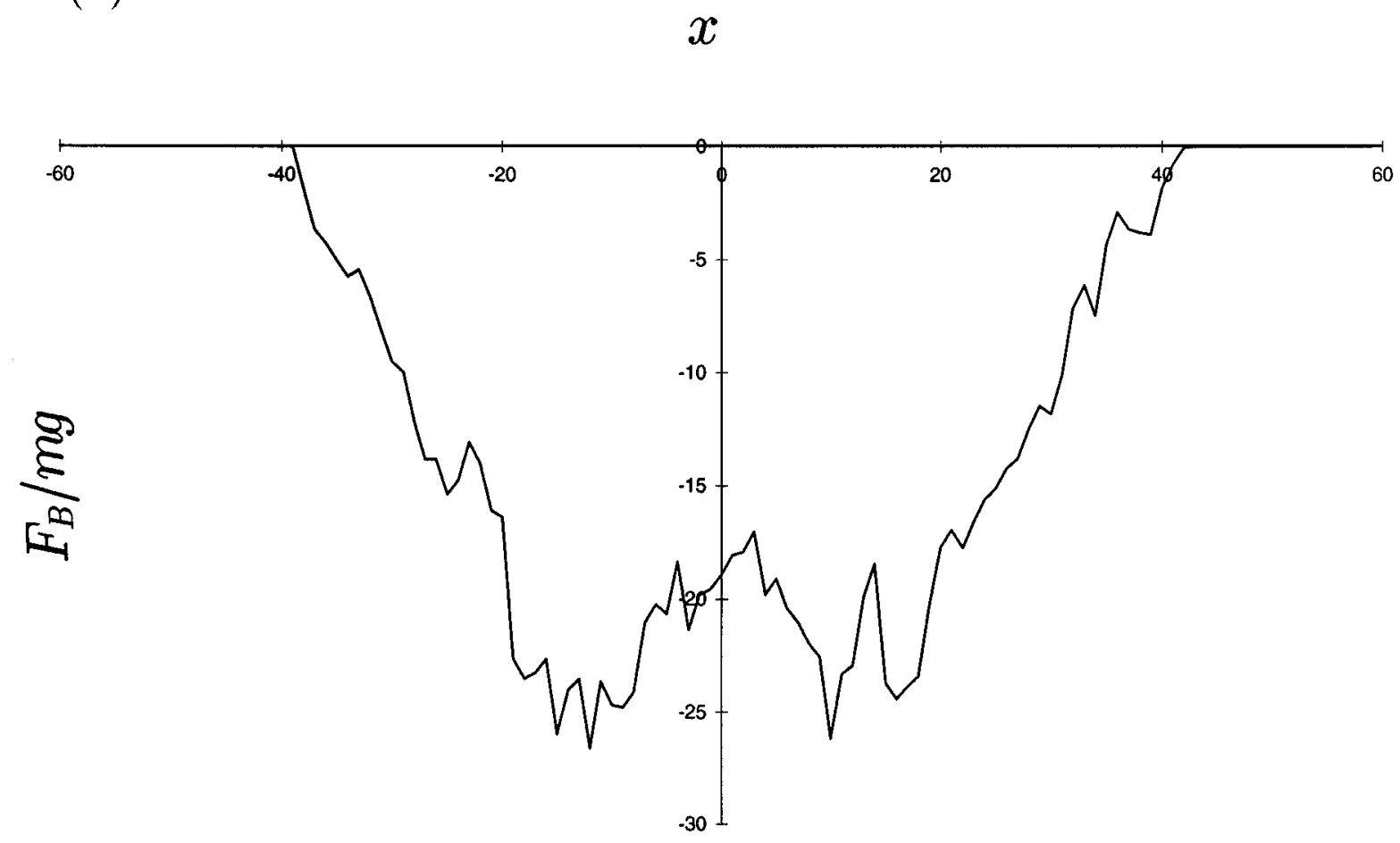

FIG. 3. (a) Normal force vectors for the configuration shown in Figs. 1 and 2(d) and (b) the normal force distribution on the base $F_{B} / m g$.

total force between granules $i$ and $j$, denoted by $\mathbf{F}_{i j}$, is the sum of normal $\mathbf{F}_{i j}^{N}$ and tangential $\mathbf{F}_{i j}^{T}$ components

$$
\mathbf{F}_{i j}=\mathbf{F}_{i j}^{N}+\mathbf{F}_{i j}^{T}
$$

The total force on granule $i$ is

$$
\mathbf{F}_{i}=\sum_{k=1}^{n_{c}} \mathbf{F}_{i k}+\mathbf{F}_{i}^{g}
$$

where the sum is over all the contacts $n_{c} . \quad \mathbf{F}_{i}^{g}$ is the gravitational force acting on granule $i$. Both the normal and the tangential force consist of a term that depends on position or accumulated displacement and a velocity-dependent force representing contact damping. For the normal direction we have

$$
\mathbf{F}_{i j}^{N}\left(r_{i j}, \dot{\mathbf{r}}_{i j}\right)=\left[F_{e}^{N}\left(r_{i j}\right)-C_{N}\left(r_{i j}\right) \dot{\mathbf{r}}_{i j} \cdot \hat{\mathbf{n}}\right] \hat{\mathbf{n}},
$$

where $\hat{\mathbf{n}}=\left(\mathbf{r}_{i j} \cdot \mathbf{x}_{i j}, \mathbf{r}_{i j} \cdot \mathbf{y}_{i j}\right) / r_{i j}$ is the normal unit vector for the contact. There is also a velocity-dependent dissipative force (to reflect the inelastic nature of real contact interactions when in relative motion) that is based on the solution of a classical damped harmonic oscillator, with force constant $k$. For the normal interaction, the damping force is the product of a damping coefficient $C_{N}(r)$ times the instantaneous relative velocity component in the normal direction. $C_{N}(r)$ was chosen to be an arbitrary fraction $(50 \%)$ of the critical damping coefficient $C_{c}=2[m k(r)]^{1 / 2}$, where $k(r)$ $=-d F_{e}^{N}(r) / d r$.

Similarly, in the sliding or "tangential" direction, defined for each contact in terms of the current center-to-center vector $\mathbf{r}_{i j}$, we have 


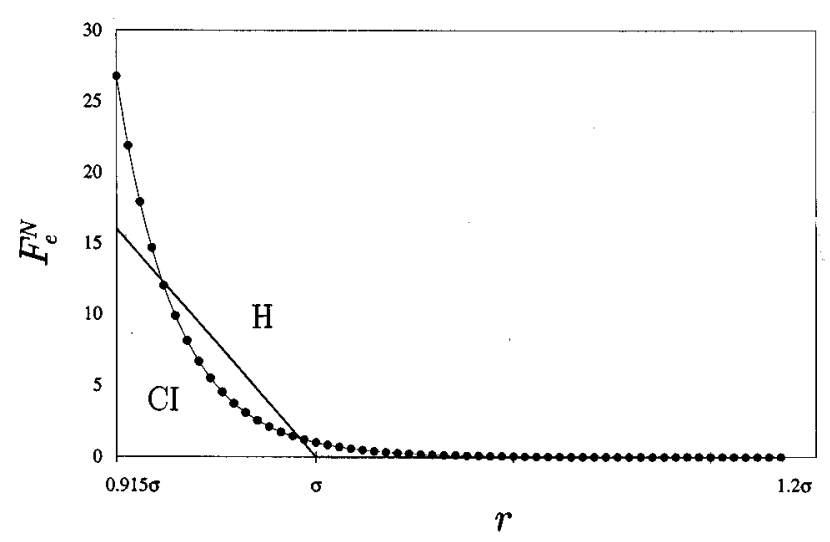

FIG. 4. Comparison between the continuous interaction force law and the equivalent Hooke law form, which gives rise to equal work done in a contact up to $r_{i}$. Key: H, Hooke's law; CI, continuous interaction with $n=36$ and $r_{i}=0.915$.

$$
\mathbf{F}_{i j}^{T}\left(\delta_{F}, \dot{\mathbf{r}}_{i j}\right)=\mathbf{F}_{e}^{T}\left(\delta_{F}\right)-C_{T}\left(\delta_{F}\right)\left(\dot{\mathbf{r}}_{i j} \cdot \hat{\mathbf{t}}\right) \hat{\mathbf{t}}
$$

and

$$
\mathbf{F}_{e}^{T}\left(\delta_{F}\right)=\mu F_{e}^{N}\left[1-\left(1-\frac{\left|\delta_{F}\right|}{\delta_{\max }}\right)^{3 / 2}\right] \hat{\mathbf{t}},
$$

where $\mu$ is the friction coefficient (typically $\mu \sim 0.4-0.6$ ) and $\hat{\mathbf{t}}=\left(\mathbf{r}_{i j} \cdot \mathbf{y}_{i j},-\mathbf{r}_{i j} \cdot \mathbf{x}_{i j}\right) / r_{i j}^{2}$ is the tangential unit vector. The contact displacement $\delta_{F}$ is a cumulative scalar quantity representing the distortion of the contact zone arising from the relative movement of two disks in contact caused by a combination of rolling and sliding, with the form

$$
\delta_{F}=\int_{0}^{t}\left\{\dot{\mathbf{r}}_{i j}(s) \cdot \hat{\mathbf{t}}(s)-\left[\dot{\theta}_{i}(s)+\dot{\theta}_{j}(s)\right] r_{i j}(s)\right\} d s,
$$

where the time variable $s=0$ is when the two particles first come into contact or "engage" at a separation $r_{i j}=r_{0}$ and subsequently gives rise to a normal deformation $\delta_{N}=r_{0}-r>0$ as the particles come closer together. We have chosen the value $r_{0}=1.2 \sigma$, which corresponds to a cutoff force of $10^{-3} \mathrm{mg}$ for the $n=37$ force law. This extended region of low load engagement is a crucial element of the model, reflecting the significance (as discussed above) of the "assembly deformation" element of the true physics.

Note that $\delta_{F}$ arises from the sum of the angular velocities of the two disks in contact. Two disks rotating in the opposite sense with the same angular velocity do not give rise to a relative displacement of the two surfaces in the contact zone. Also hysteresis in the tangential displacement resulting from different loading and unloading paths is ignored. The analytic form of the tangential frictional interaction is not yet known, but the expression in Eq. (5) has reasonable limiting values. It has a resistance force that is linear in $\delta_{F}$ for $\delta_{F} / \delta_{\text {max }} \ll 1$, i.e., an elastic response region. As $\delta_{F} \rightarrow \delta_{\max }$ the compliance increases and the frictional force tends to Amonton's law, i.e., $F_{e}^{T}=\mu F_{N}$. If the mechanism of surface deformation in the normal and tangential directions is similar, it is reasonable to assume proportionality between the average normal and tangential moduli, i.e., the value of the average tangential modulus up to gross sliding $\left\langle d \mathbf{F}_{e}^{T} / d \delta_{F}\right\rangle \sim F_{e}^{N} / \delta_{N}$. From this follows a proportionality relationship between normal and maximum tangential displacements of the form $\delta_{\max }=\alpha \mu \delta_{N}$, where $\alpha$ is a constant that depends on the analytic form of $\mathbf{F}_{e}^{T}\left(\delta_{F}\right)$. (It is interesting to note that a Hertzian analysis for normal interactions coupled with Mindlin's frictional analysis also gives the same linear relationship [1].) To be consistent with our previous studies we have used $\alpha=1.214$, which is based on material property considerations for typical elastic contacts [1]. The general result that $\delta_{\max }=\alpha \mu \delta_{N}$ does not rely upon the use of a constant compliance; it follows from consideration of the average contact stiffness in the tangential direction $\left\langle d \mathbf{F}_{e}^{T} / d \delta_{F}\right\rangle$. Provided that $F_{T}$ reaches a maximum, constant value $F_{T}=\mu F_{N}$ at $\delta_{F}=\delta_{\max }$, and $F_{T}=0$ when $\delta_{F}=0$, the result holds for any functional form of $\delta_{F}$. The typical values of $\delta_{\max }$ observed in our simulations are $\sim(0.1-0.2) \sigma$, which are several orders of magnitude larger than would be the case for a single point contact between elastic spheres of realistic elastic contacts (e.g., for seeds or sand).

Although the analytic form of the relationships between normal and tangential displacements might be construed as coming from the classical theory of surface interactions, i.e., Mindlin and Hertz theory, the interactions used in this model represent quite different physics on a much larger length scale. In modeling surface displacements of single particleparticle contacts, a load-independent contact stiffness is used, e.g., Hooke's law constant, which arises from the material's Young modulus and Poisson ratio. In contrast, when modeling relative displacements of individual particles in an evolving assembly, the stiffness of the particle matrix is a strong function of the local voidage (i.e., the assembly deformation), which in turn is related to the separation between particle centers. Hence we require a displacement-dependent matrix stiffness, which is offered by the continuousinteraction model. The incorporation of assembly aspects into the pair interaction gives rise to effective bulk moduli, which are much softer than that of the solid material, and typical maximum tangential displacements, which are much larger [1].

The tangential stiffness $k_{T}$ is found by differentiating Eq. (5), giving

$$
k_{T}\left(\delta_{F}\right)=\frac{d \mathbf{F}_{e}^{T}\left(\delta_{F}\right)}{d \delta_{F}}=\frac{3 \mu F_{N}}{2 \delta_{\max }}\left(1-\frac{\left|\delta_{F}\right|}{\delta_{\max }}\right)^{1 / 2},
$$

and as for the normal direction, we adopt a damping coefficient that is $50 \%$ of the critical damping value $C_{\mathrm{cr}}=2\left[m k_{T}\left(\delta_{F}\right)\right]^{1 / 2}$. Thus the tangential damping coefficient in Eq. (4) is given by

$C_{T}\left(\delta_{F}\right)=\left[m k_{T}\left(\delta_{F}\right)\right]^{1 / 2}=\left[\frac{3 m \mu F_{N}}{2 \delta_{\max }}\left(1-\frac{\left|\delta_{F}\right|}{\delta_{\max }}\right)^{1 / 2}\right]^{1 / 2}$.

This is reasonable as maximum damping is likely to be at a maximum just after contact formation, i.e., $\delta_{F} \ll \delta_{\max }$.

The position and angular velocity update schemes are

$$
\begin{aligned}
& \mathbf{r}_{i}(t+\Delta t)=\mathbf{r}_{i}(t)+\dot{\mathbf{r}}_{i}(t+\Delta t / 2) \Delta t, \\
& \theta_{i}(t+\Delta t)=\theta_{i}(t)+\dot{\theta}_{i}(t+\Delta t / 2) \Delta t,
\end{aligned}
$$

where 
(a)

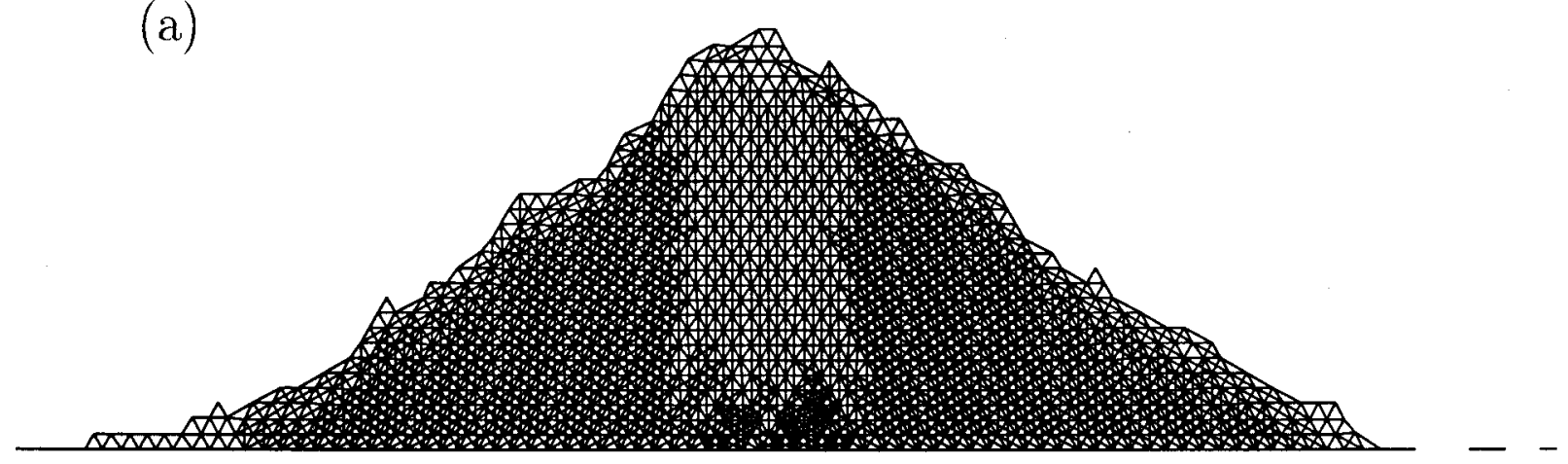

(b)

$x$

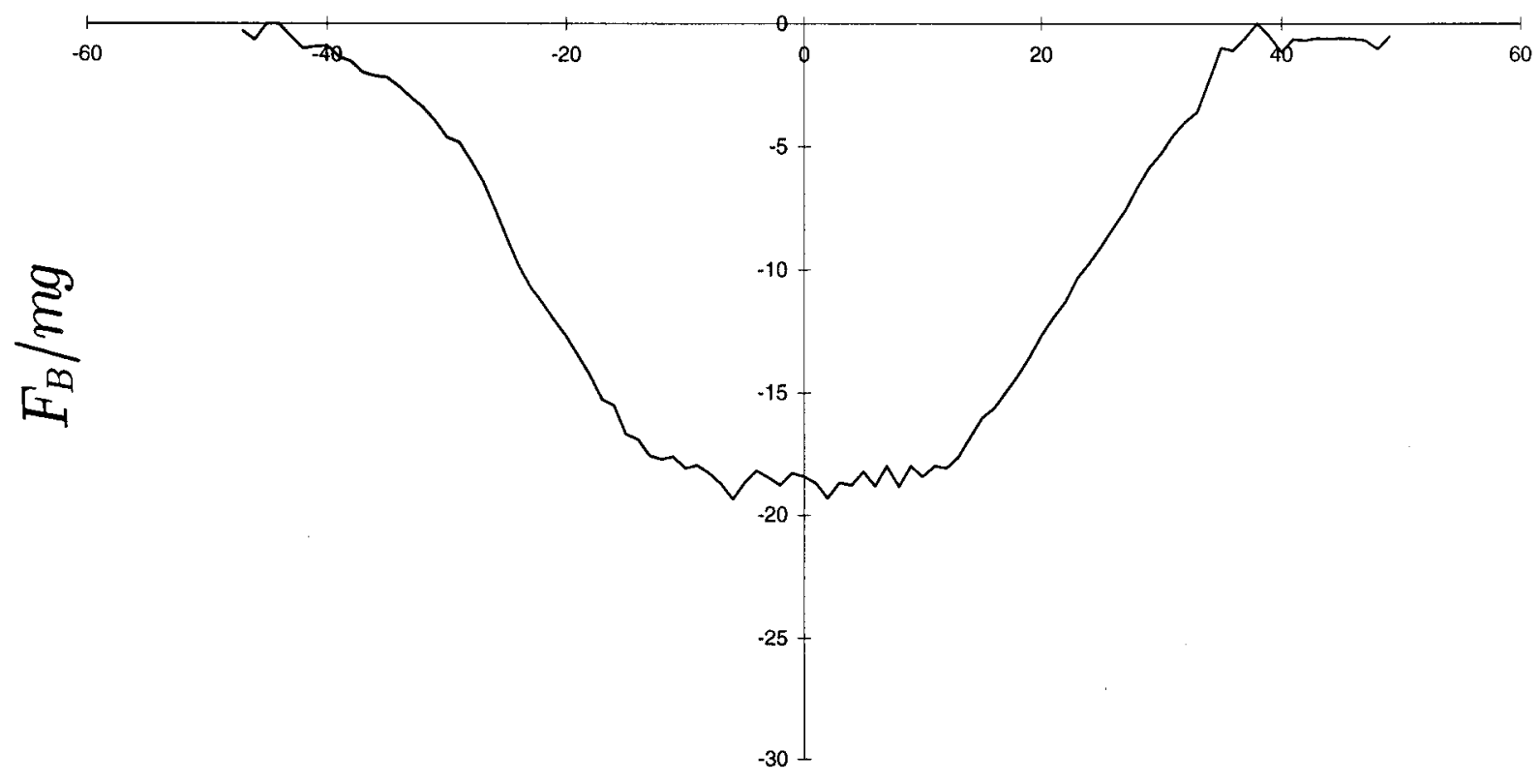

FIG. 5. (a) Density distribution of a stable heap of 1000 monodisperse particles represented by a linear spring normal interaction with force constant $k=500$. (b) Corresponding normal force distribution on the base from the structure.

$$
\begin{gathered}
\dot{\mathbf{r}}_{i}(t+\Delta t / 2)=\dot{\mathbf{r}}_{i}(t-\Delta t / 2)+\mathbf{F}_{i}(t) \Delta t / m, \\
\dot{\theta}_{i}(t+\Delta t / 2)=\dot{\theta}_{i}(t-\Delta t / 2)+T_{i}(t) \Delta t / I,
\end{gathered}
$$

where $T_{i}$ is the torque on granule $i$ and $I=m \sigma^{2} / 8$ is the moment of inertia of the disk,

$$
T_{i}(t)=\sum_{k=1}^{n_{c}} \mathbf{r}_{i j} \times \mathbf{F}_{i j}
$$

The simulation time step was typically $0.001 \sigma^{1 / 2} g^{-1 / 2}$. All quantities are reported in reduced units of $\sigma$ for distance, $(\sigma g)^{1 / 2}$ for velocity, $g$ for acceleration, and $m g$ for force. In some of the simulations presented in this work, the disks were all the same size. In others, the disks were polydisperse, i.e., there was a distribution of disk sizes. In the latter case, the samples were fully polydisperse in that there was a continuous distribution of particle sizes. Percentage polydisper- sity was defined such that, for a $\delta \%$ polydisperse sample, the size of each disk was randomly chosen from a uniform distribution in the range $(1-\delta) \sigma \rightarrow \sigma$. Thus the mean particle size was approximately $(1-0.5 \delta) \sigma$ with an approximate standard deviation of $0.25 \delta \sigma$. Simulation results for $\delta=0 \%$ (monodisperse), $\delta=5 \%$, and $\delta=10 \%$ are presented in this paper.

The simulation procedure was to allow the grains to fall under gravity in the $y$ direction onto the substrate from an initial height of $\sim 100 \sigma$ with initial lateral $x$ coordinates chosen from a uniform random distribution in an interval $\pm 3 \sigma$. In the preliminary stages of this work attempts were made to deposit the grains onto a flat smooth structureless surface interacting with the particles with an $m g(\sigma / y)^{37}$ force. However, owing to the low vertical forces and high kinetic energy of the grains on contact, a stable heap could not be formed. Stable heaps were formed on a static substrate consisting of a row of equally spaced particles, interacting with the dynamic grains with the same interaction laws. 
(a)

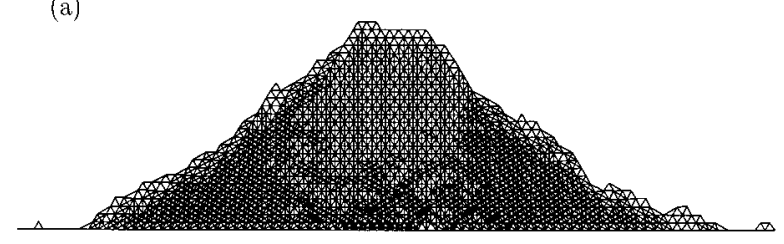

(b)

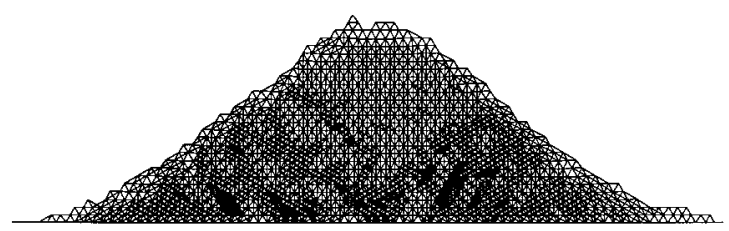

FIG. 6. Density distribution for stable heap of 1000 monodisperse particles for an $n=144$ simulation with the interaction truncation point (a) $r_{0}=1.047 \sigma$ and (b) $r_{0}=1.2 \sigma$.

\section{RESULTS AND DISCUSSION}

Figure 1 shows the final rest state of 1334 monodisperse disks deposited on the structured substrate. The growth of the heap took place by a series of avalanches in a boundary layer 3-4 particles thick on the surface, with the inner particles largely static during this process; this behavior is consistent with experimental observation (e.g., [10]). The angle of repose (the angle between the inclined surface and the base) is $35^{\circ} \pm 5^{\circ}$. The angle of repose was found to decrease with increasing substrate particle spacing $S$, with a local plateau in the range $S \gtrsim 1.1 \sigma$, where the angle was insensitive to the spacing. This value of $S$ corresponds to loose random packing in two dimensions with a surface coverage of 0.76 . The figure shows two vacancy defects, dislocations, and some semicrystalline ordering for several layers near the base. [This is more clearly illustrated in Fig. 1(b) for filled in disks.] The mechanical stability of $60^{\circ}$ pyramidal heaps formed by placing the particles in a triangular lattice on the base was investigated. These were found to be unstable and partially collapsed at the boundaries to form buttresses, which stabilized the remaining core of the original structure.

The heap evolves by means of a series of discrete avalanches. In Fig. 2 we show the evolution of this heap, capturing the formation of a mechanically unstable surface structure [Fig. 2(a)] and the resulting avalanche [Fig. 2(b)]. The method of presentation is different from Fig. 1, in that we show vectors between the disk centers, which illustrate the network structure and the density distribution more effectively. Lighter regions on the figure indicate high voidage. An avalanche is apparent as an open framework on the righthand side of Fig. 2(b), indicating that the density at the surfaces of the heap during an avalanche is much lower than at other places. The interior of the heap is largely unaffected during an avalanche; the avalanche is confined to a boundary layer near the surface. The effect of a series of avalanches is to reduce irregularities in the local angle of repose on the heap surface. For example, the right-hand surface in Fig. 2(a) shows considerable variation in the local angle of repose, whereas after the avalanche there is an almost uniform slope along the whole length of the surface. The central region of the heap, around halfway between the apex and the base, has a low density (lighter shading), indicating a higher voidage with a patchwork of compacted regions formed close to the base. The packing density is highly nonuniform throughout the heap. In general, regions near the base of the heap are most densely packed, with looser packing towards the edges and apex.

The contact force distribution on the base reflects the internal structure of the heap. It might be expected that the highest normal force on the base would appear below the apex, but this is not the case, as a result of the relatively low internal contact forces in the central region of the heap. This $\mathrm{M}$ profile of base force has been found in simple block models of heaps $[11,12]$ and in experimental measurements [13] of the normal stress across the base of the midplane of a three-dimensional (3D) heap. Although we are considering a 2D system and the experiments were performed on 3D heaps, the results are qualitatively very similar. We believe the axial symmetry conferred by the conical shape of the experimental heap to be significant. Previous work [1,2] indicates that $2 \mathrm{D}$ results largely agree with those from the midplane of an axially symmetric 3D arrangement.

The weight of the heap in the model system studied here is to some extent transmitted away from the part of the base below the apex, as seen in Fig. 3(b), which shows the normal force vectors within the heap. The normal forces form lines of principal stress extending outward from points on the upper surface of the heap towards the base, with a higher angle of inclination to the base than the heap itself. Components of normal force in other directions are very small. Lines of principal stress intersect at a point below the apex to form an arch, immediately beneath which the internal stresses are somewhat smaller. Figure 3(a) shows that the internal force distribution is reflected in a local minimum of base normal force on the portion below the arch, with a maximum on each side. The maximum force is some 20-30\% greater than that in the center of the base. The maxima in base normal force correspond with the intersection with the base of the longest lines of principal stress.

In order to assess the effect of the analytic form for the excluded-volume component of the normal force $F_{e}^{N}$, we have performed simulations with a simple Hooke law spring interaction: $F_{e}^{N}=k(\sigma-r)$ for $r<\sigma$ and $F_{e}^{N}=0$ for $r>\sigma$. The value of the spring constant $k$ was an average, apparent Hooke law constant derived from the continuous interaction on an equivalent work performed basis over the typical range of overlap observed in a simulation. The minimum separation $r_{i}$ was found from the simulation data, and the equivalent Hooke law constant was calculated by equating areas below the two analytic forms as indicated in Fig. 4. The formula is

$$
k_{\text {equiv }}=\frac{2 \int_{r_{0}}^{r_{i}} F_{e}^{N}(r) d r}{\left(\sigma-r_{i}\right)^{2}} .
$$

The density distribution map for a Hooke law simulation using a monodisperse system is given in Fig. 5(a). The distribution of voidage is quite different from the comparable continuous interaction case [Fig. 2(d)]. There is a large region of low voidage below the apex that is surrounded on the wings of the heap by moderately dense material. There is a small area of high compaction just near the base below the 


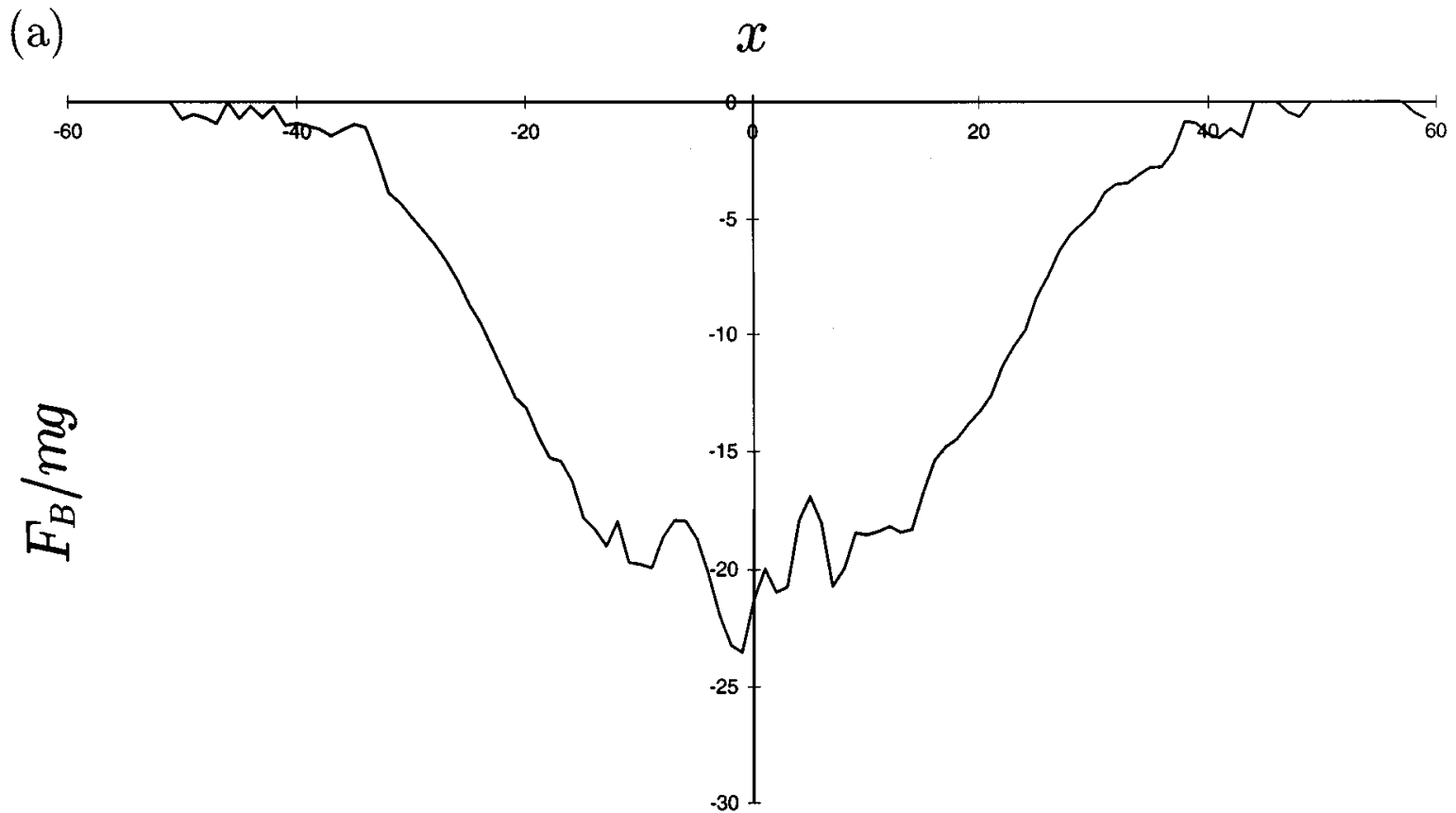

(b)

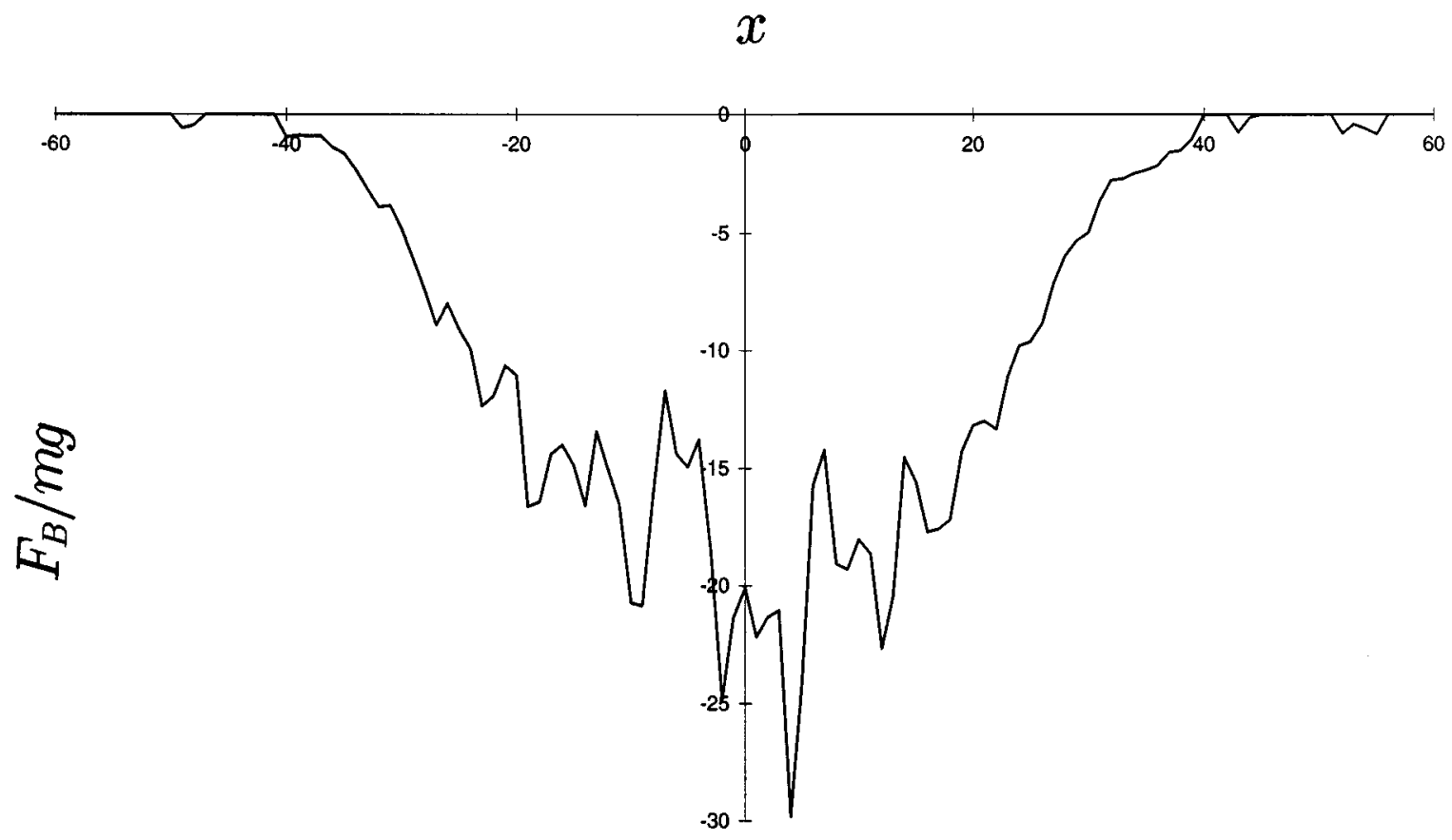

FIG. 7. Normal force distribution on the base $F_{B} / m g$, from the structure shown in (a) Fig. 6(a) and (b) Fig. 6(b).

apex. The corresponding base stress distribution shown in Fig. 5(b) has lost the bimodal feature. In this simulation, heap evolution in response to continual feeding appears to take place by small numbers of grains rolling down the sides of the growing structure; large-scale avalanches are not observed. Therefore, the matrix shows little evidence of assembly cohesion and collective dynamics with this short-range interaction.

Many of the results presented in this paper are from $n=36$ simulations. The evidence shows that simulations using this value of the index are particularly useful in highlighting long-range bulk shear phenomena. However, it was found that particle overlaps under the highest normal loads in the heaps were rather large (several percent of the particle diameter). Thus it is necessary to consider stiffer particle-particle interactions. The effect of the continuous interaction index $n$ has been assessed by performing simulations of monodisperse particle samples using a considerably steeper interaction with $n=144$. The interaction for $n \neq 36$ was truncated such that the force at the cutoff point was equal; for the $n=144$ simulations, this was $r_{0}=1.047 \sigma$. The density distribution for such a simulation is shown in Fig. 6(a). Varying 
(a)

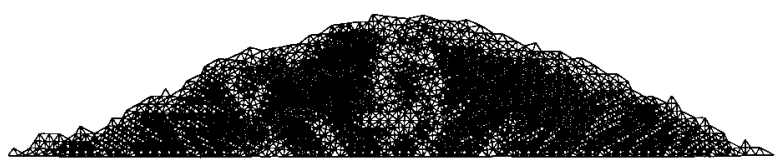

(b)

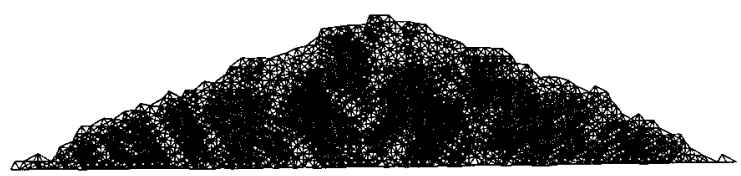

FIG. 8. Density distributions for stable heaps of 1000 polydisperse particles with (a) $5 \%$ uniform polydispersity at $2.35 \times 10^{3}$ time units and (b) $10 \%$ uniform polydispersity at $1.75 \times 10^{3}$ time units.

the index $n$ appears not to affect greatly the shape of the heap; the angle of repose was $35^{\circ}$ as opposed to $31^{\circ}$ for the $n=36$ case. However, there are clear differences in the density distribution; the "shear banding", seen in $n=36$ simulations (e.g., Fig. 2) largely disappears. The distribution shown in Fig. 6(a) more closely resembles that for the equivalent Hooke law model shown in Fig. 5(a). This is unsurprising given that as the index $n$ increases, the continuous interaction model closely resembles a Hooke law form; in the limit of infinite steepness in both cases the two are identical.

As discussed above, we consider assembly deformation to be crucial in understanding the mechanics of many-body granular systems. The significance of assembly deformation is manifest in the length of the tapered region of the interaction, i.e., the portion of the interaction beyond $r=\sigma$. We have assessed the importance of this by performing a simulation maintaining $n=144$, but extending the interaction tail by using the original truncation point $r_{0}=1.2 \sigma$. The effect is quite significant, despite the fact that the normal interaction force $F_{e}^{N}$ is very low in the region $1.047 \sigma<r<1.2 \sigma$. The density distribution for this simulation is shown in Fig. 6(b), and shows shear banding. The base normal force distributions for the two $n=144$ simulations are shown in Figs. 7(a) and 7(b). The distribution for the short-tail simulation closely resembles that for the equivalent Hooke law simulation [Fig. $5(\mathrm{~b})$ ], and while neither distribution displays a clear M profile as does the $n=36$ simulation [Fig. 3(b)], the distribution for the extended tail simulation is closer to experiment.

Small levels of polydispersity have pronounced effects on the voidage and force distributions. Figure 8 shows the rest configurations for 1000 particle systems containing 5\% [Fig. $8(\mathrm{a})]$ and $10 \%$ [Fig. 8(b)] polydispersity. These are to be compared with Fig. 2(d), the analogous $0 \%$ polydisperse case. The heaps are flatter and show much higher levels of compaction (the dark patches). The lighter low compaction regions are now more randomly distributed within the heap; there is little shear banding. The degree of compaction increases with polydispersity. There appear to be clear boundaries between low-density areas of the heap (mostly near the surface) and the compacted regions in the interior. This is in contrast with the distributions for monodisperse heaps, which in general show more gradual transitions between regions of differing density. The force distribution on the base shows greater fluctuation than in the monodisperse case; peaks in the force correspond to the intersection with the base of the high-stress compacted regions in the heap. Although such small levels of polydispersity are sufficient to affect the macrostructure of the heap, they are insufficient to produce particle segregation. The heap properties were found to be relatively insensitive to the value of the friction coefficient in the sensible range $0.2<\mu<0.6$. This indicates that heap properties and stability are dominated by geometrical criteria. However, there is some sensitivity to the analytic form of the configuration component of the normal interaction.

\section{CONCLUSION}

We have established the methodology required to form free-standing 2D heaps by granular dynamics. A continuous interaction law between the particles (which can be regarded as a separation-dependent linear spring) gives a more realistic description of granular heaps than a Hooke law model. Experimentally verified features of granular heaps ( $\mathrm{M}$ profiles of normal force on the base and shear banding) are far more apparent in simulations using the continuous interaction. Furthermore, a tapered long-range region of this interaction, which governs the details of assembly deformation, is required to reproduce many of the features of granular material in heaps. A parameter exploration was carried out that showed a sensitivity to a relatively small level of polydispersity (which is necessary to break up quasicrystalline structures and mechanics). However, the results were insensitive to the value of the contact friction coefficient, indicating that the heap properties are dependent on geometry on a length scale at or above that of the particle diameter rather than the contact-zone scale of two interacting particle surfaces. It appears that the contact friction interaction is only required to " "pin', the particles together with an adequate degree of endurance, but the assembly behavior is relatively insensitive to its analytic details. The GD technique used in the work presented in this paper is especially useful as it allows us to relate the base normal force to the internal structure and stresses in the pile.

\section{ACKNOWLEDGMENTS}

J.B. thanks the Biotechnology and Biological Sciences Research Council of Great Britain for financial support. Useful discussions with Dr. K. Wojciechowski and A. Branka of the Institute of Molecular Physics, Poznan, Poland are gratefully acknowledged.
[1] P. A. Langston, U. Tüzün, and D. M. Heyes, Chem. Eng. Sci. 50, 967 (1995).

[2] P. A. Langston, U. Tüzün, and D. M. Heyes, Powder Technol. 85, 153 (1995).
[3] O. R. Walton, Mech. Mater. 16, 239 (1993).

[4] T. Pöschel, J. Phys. (France) II 3, 27 (1993).

[5] O. Pouliquen and R. Gutfraind, Phys. Rev. E 53, 552 (1996).

[6] R. E. Snyder and R. C. Ball, Phys. Rev. E 49, 104 (1994). 
[7] L. Verlet, Phys. Rev. 159, 98 (1967).

[8] P. A. Thompson and G. S. Grest, Phys. Rev. Lett. 67, 1751 (1991).

[9] R. D. Mindlin and H. Deresiewicz, J. Appl. Mech. Trans. ASME 20, 327 (1953).

[10] H. M. Jaeger, S. R. Nagel, and R. P. Behringer, Phys. Today
49 (4), 32 (1996).

[11] E. Li and D. F. Bagster, Adv. Powder Tech. 3, 129 (1992).

[12] E. Li and D. F. Bagster, Adv. Powder Tech. 3, 137 (1992).

[13] J. Smid and J. Novosad, Inst. Chem. Eng. Symp. Ser. 63, DV/ v/1 (1981). 